\title{
Etiology of Non-Traumatic Seizures in Children Admitted to PICU: An Eight-Year Retrospective Study
}

\section{Xiaolu Deng \\ Fang $\mathrm{He}$ \\ Ciliu Zhang \\ Fei Yin \\ Jing Peng}

Department of Pediatrics, Xiangya Hospital, Central South University,

Changsha, People's Republic of China
This article was published in the following Dove Press journal: International Journal of General Medicine

Purpose: Seizure is a serious neurological symptom in the pediatric intensive care unit (PICU), yet data on etiologies of non-traumatic childhood seizures in China are scarce. We aimed to investigate the etiological trends of seizures in children admitted to the PICU in our tertiary center. Patients and Methods: We performed a retrospective analysis of all patients (aged 29 days to 14 years) with non-traumatic seizures, admitted to the PICU of Xiangya Hospital from 2010 to 2017. Etiological analysis was performed to compare data between 2010-2013 and 2014-2017.

Results: The study included 318 patients (male: female $=1.27: 1$ ) with mean age of $5.4 \pm 4.0$ years. The most frequent causes observed were CNS infections (109/318, 34.3\%), unknown reason epilepsy $(96 / 318,30.2 \%)$, and immune $(56 / 318,17.6 \%)$. Comparison of the 2010-2013 and 2014-2017 periods revealed a significant decrease in the percentage of CNS infections [48.8\% (59/121) vs 25.4\% (50/197), p < 0.001] and a significant increase in the incidence of unknown reason epilepsy [(24/121, 19.8\%) vs $(72 / 197,36.5 \%)$, p < $0.001]$ and immune causes [(12/121, 9.9\%) vs $(44 / 197,22.3 \%), \mathrm{p}=0.005]$.

Conclusion: CNS infections were the most common cause of seizures during 2010-2013, while non-infectious diseases such as epilepsy and immune disorders represented the leading causes during 2014-2017. Continuous improvement in medical technology and understanding of disease patterns would greatly improve early diagnosis and therapeutic management of such conditions.

Keywords: epilepsy, autoimmune encephalitis, CNS infections, pediatric

\section{Introduction}

Seizures are the most common medical problem for emergency medical services transport in children, accounting for approximately $15 \%$ of all pediatric calls in the USA. ${ }^{1}$ Pediatric status epilepticus is associated with a short-term mortality of $0-3 \%$ and long-term mortality of approximately $7 \%{ }^{2}$ Children admitted to the PICU show significant differences in etiology compared with adults and those in general pediatric wards. However, there have been limited population-based and hospital-based studies of pediatric groups with seizures. A pediatric study showed the most common causes of seizures in a PICU setting were acute symptomatic in $53.3 \%$ and epileptic in $46.7 \%$ of patients. ${ }^{3}$ Acute symptomatic seizures are caused by an acute event such as stroke, traumatic brain injury (TBI), or CNS infection. Early recognition of potentially life-threatening conditions and proper management are, therefore, vital for favorable outcomes.

Various etiological factors have been identified for seizures. CNS infections initially received much attention; however, growing understanding of immune, epileptic, and genetic causes are likely to increase incidence figures because
Correspondence: Jing Peng

Department of Pediatrics, Xiangya Hospital, Central South University, No. 87, Road

Xiangya, Changsha, Hunan 410008, People's Republic of China

$\mathrm{Tel}+86$ I3548966986

$\mathrm{Fax}+86731-84327922$

Email pengjing627@।26.com
International Journal of General Medicine 2020:13 |285-1290 
available diagnostic tests such as auto-antibody testing and genetic testing were limited in the earlier period, especially in a developing country. A study by the California Encephalitis Project showed that the frequency of AntiN-methyl-D-aspartate receptor (NMDAR) encephalitis surpassed that of any individual viral etiology in young individuals. ${ }^{4}$ Genetic sequencing has been increasingly used in clinical diagnostics for a variety of indications to identify the underlying genetic cause of diseases for which seizures may be the first and major manifestation.

The study of the epidemiology in terms of etiology and time trends is important to aid further understanding of patterns of the disease, gain useful information for future research, and aid priority setting in the prevention and treatment of encephalitis. The objective of this study was to review the etiology of non-traumatic seizures in patients admitted to the PICU at a university hospital in China and describe the long-term trends in diagnosis over eight years.

\section{Patients and Methods}

Data were collected from the medical records (including first page, progress notes, examination reports, and discharge record) and exported from the Hospital Information System (HIS) in the Department of Pediatrics of Xiangya Hospital (tertiary hospital, teaching hospital of Central South University), Changsha, China. The inclusion criteria were as follows: (1) patients presented with seizure; (2) required intensive care services; (3) admission time between January 1, 2010, and December 31, 2017; and (4) children were 29 days to 14 years of age (ie, the neonatal period was excluded). Medical records that did not meet these criteria were deemed ineligible for the current study. Exclusion criteria: A previous traumatic event in the period of 3 month or less before the current event.

The following information was obtained from the medical records of each patient: age, sex, primary complaint, birth history, developmental history, family history, laboratory test results (white blood count, C-reactive protein, erythrocyte sedimentation rate (ESR), serum electrolytes, blood sugar, lactic acid, blood ammonia, and cerebrospinal fluid (CSF) analysis), video-electroencephalogram (EEG), cranial neuroimaging (CT scan and/or magnetic resonance imaging), and final diagnosis. Suspected autoimmune encephalitis in patients typically resulted in CSF and serum studies, including paraneoplastic and autoimmune antibody panels, and serum studies, including thyroid antibodies, antinuclear antibody, anti-double-stranded DNA, and anti-SSA/SSB autoantibodies. Samples from patients with suspected genetic, metabolic, or epileptic diseases were subjected to specific gene panels, whole-exome sequencing (WES), and gas chromatography-mass spectrometry (GC-MS) testing.

Etiology was classified into the following categories: CNS infections, unknown reason epilepsy, immune, metabolic, genetic, toxic, vascular, structural, posterior reversible encephalopathy syndrome (PRES), degenerative, endocrine, and unknown. Diagnoses were made based on recorded clinical and laboratory data and verified with standard references. Significant differences in diagnoses between the 2010-2013 and 2014-2017 groups were evaluated using Fisher's exact tests. The threshold for significance was $\mathrm{p}<0.05$. Uncommon etiologies (those that make up less than $3 \%$ of the population) group into one "Other" category. The data for all patients were put into a data bank using Microsoft Excel. The same program was used for calculations. The institutional review board approved the present study as a clinical observational study; thus, ethics committee approval was not required.

\section{Results}

There were 318 patients (males, 178; females, 140) included in this study, with a mean age of $5.4 \pm 4.0$ years (Table 1). The three most common causes of nontraumatic seizures observed were CNS infections (109/ 318, 34.3\%), unknown reason epilepsy (96/318, 30.2\%), and immune $(56 / 318,17.6 \%)$. The rest of etiologies (Figure 1) include metabolic (16/318, 5.0\%), genetic (11/ $318,3.5 \%)$, toxic $(9 / 318,2.8 \%)$, vascular $(8 / 318,2.5 \%)$,

Table I Patient Characteristics $(n=318)$

\begin{tabular}{|l|l|}
\hline No. of Cases & 318 \\
\hline $\begin{array}{l}\text { Gender } \\
\text { Male }\end{array}$ & $178(56 \%)$ \\
Female & $140(44 \%)$ \\
\hline Male/Female ratio & $1.27: 1$ \\
\hline Age group (years) & \\
$<1 y$ & $40(13 \%)$ \\
$1-3 y$ & $79(25 \%)$ \\
$3-6 y$ & $79(25 \%)$ \\
$6-12 y$ & $81(25 \%)$ \\
$\geq 12 y$ & $39(12 \%)$ \\
\hline Period (years) & \\
$2010-2013$ & 121 \\
$2014-2017$ & 197 \\
\hline
\end{tabular}




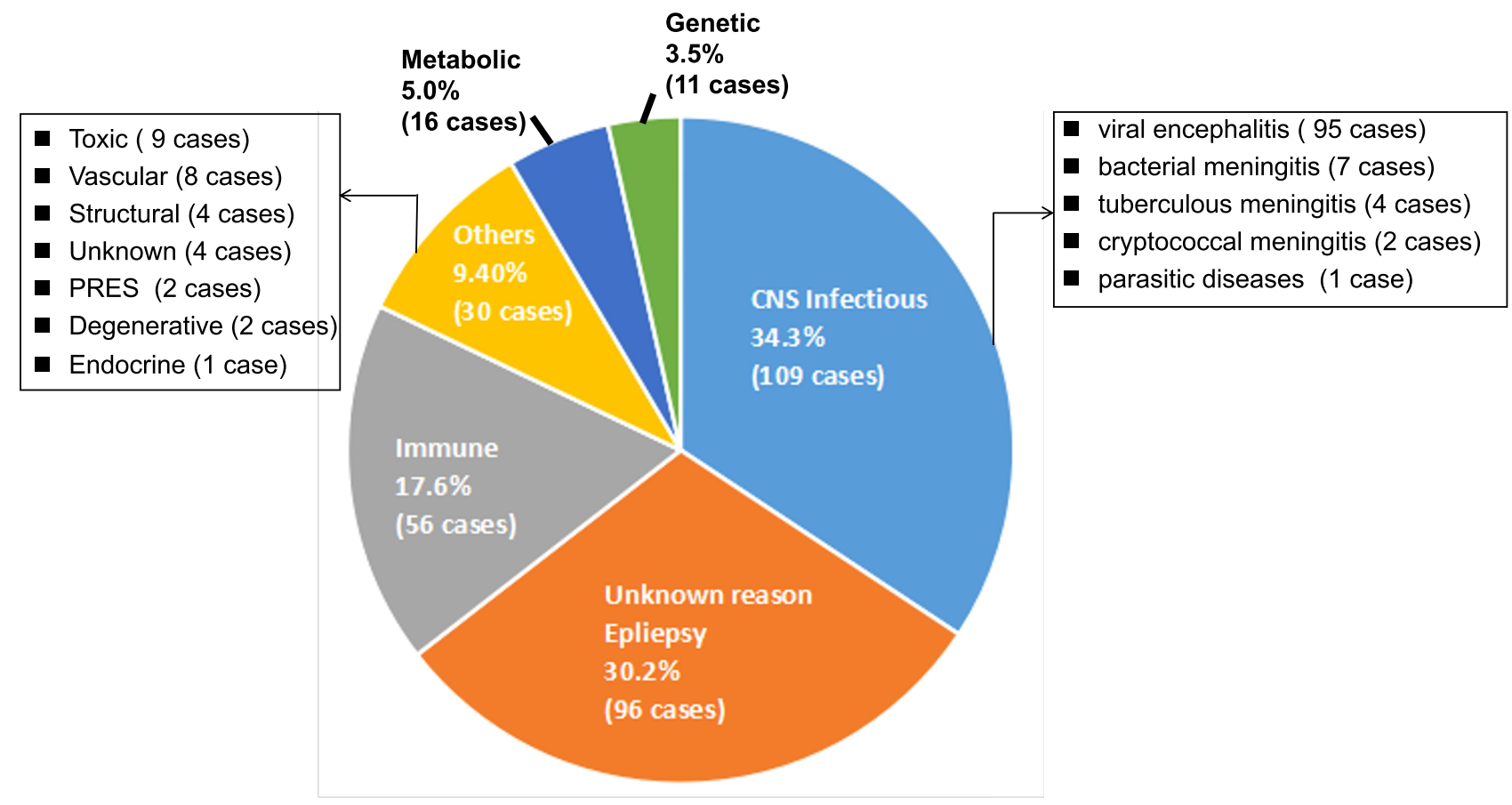

Figure I Etiology of non-traumatic seizures in children admitted to PICU $(2010-2017, \mathrm{n}=318)$.

structural $(4 / 318,1.3 \%)$, unknown $(4 / 318,1.3 \%)$, PRES $(2 / 318,0.6 \%)$, degenerative $(2 / 318,0.6 \%)$, and endocrine $(1 / 318,0.3 \%)$. Other category included Toxic, Vascular, Structural, Unknown, PRES, Degenerative and Endocrine.

CNS infections (Figure 1) included viral encephalitis $(95 / 109,87.2 \%)$, bacterial meningitis $(7 / 109,6.4 \%)$, tuberculous meningitis $(4 / 109,3.7 \%)$, cryptococcal meningitis $(2 / 109,1.8 \%)$, and parasitic diseases (1/109, 0.9\%). Genetic causes included eight cases with Dravet syndrome (seven with SCN1A mutations and one with PCDH19 mutation). There are 12 cases with Febrile infectionrelated epilepsy syndrome (FIRES) in the unknown reason epilepsy group. Immune causes included anti-NMDAR encephalitis $(33 / 56,58.9 \%)$, acute disseminated encephalomyelitis $(15 / 56,26.8 \%)$, central nervous system lupus $(5 / 56,8.9 \%)$, Hashimoto encephalopathy $(2 / 56,3.6 \%)$, and Guillain-Barré syndrome variant $(1 / 56,1.8 \%)$. All 33 cases of anti-NMDAR encephalitis were identified in the 2014-2017 period. Comparison of the 2010-2013 and 2014-2017 periods revealed a significant decrease in the percentage of CNS infections [48.8\% (59/121) vs $25.4 \%$ $(50 / 197), \mathrm{p}<0.001]$ and a significant increase in the incidence of unknown reason epilepsy [(24/121, 19.8\%) vs $(72 / 197,36.5 \%), \mathrm{p}<0.001]$ and immune causes [(12/ $121,9.9 \%$ ) vs $(44 / 197,22.3 \%), p=0.005]$ (Table 2 and Figure 2).

\section{Discussion}

Seizure etiology is an established prognostic factor of both outcome and recurrence. ${ }^{5}$ It is now well established that infectious encephalitis and immune-mediated encephalitis can present with similar clinical signs and symptoms, suggesting that prompt evaluation of seizure etiology might decrease the impact of seizures in the daily life of patients. ${ }^{6}$ There have been dramatic developments in neuroimaging methods, autoantibody detection, electroencephalographic (EEG) monitoring, and gene sequencing in recent times. In the current study, we evaluated clinical diagnoses and change of etiology in PICU patients from a tertiary hospital in China.

CNS infections are among the most common risk factor for seizures and acquired epilepsy. Most patients with encephalitis are diagnosed based on clinical manifestations and laboratory or imaging evidence of inflammation. Between 1998 and 2005, in 1570 patients with encephalitis throughout California enrolled in a study, a confirmed or probable etiologic agent was identified in $16 \%$ of the cases, and $69 \%$ of these agents were viral. ${ }^{7}$ In our study, of the 109 cases of CNS infections, $87.2 \%$ were diagnosed as viral encephalitis, which is characterized by altered mental status and various combinations of acute fever, seizures, and neurologic deficits. There are four patients with TBM were identified during 2010-2013. 
Table 2 Etiological Causes in Patients with Seizure in the 2010-2013 and 2014-2017 Periods

\begin{tabular}{|c|c|c|c|c|}
\hline Etiology & $\begin{array}{l}\text { Overall } \\
n=318 \\
(\%)\end{array}$ & $\begin{array}{l}2010-2013 \\
n=121(\%)\end{array}$ & $\begin{array}{l}2014-2017 \\
n=197(\%)\end{array}$ & $p$-value \\
\hline $\begin{array}{l}\text { CNS } \\
\text { infectious }\end{array}$ & $109(34.3)$ & $59(48.8)$ & $50(25.4)$ & $<0.001$ \\
\hline $\begin{array}{l}\text { Unknown } \\
\text { reason } \\
\text { epilepsy }\end{array}$ & $96(30.2)$ & $24(19.8)$ & $72(36.5)$ & $<0.001$ \\
\hline Immune & $56(17.6)$ & $12(9.9)$ & $44(22.3)$ & 0.005 \\
\hline Other & $30(9.40)$ & $19(15.7)$ & II (5.6) & $<0.005$ \\
\hline Metabolic & $16(5.0)$ & $6(5.0)$ & $10(5.1)$ & 0.963 \\
\hline Genetic & $11(3.5)$ & I (0.8) & $10(5.1)$ & 0.057 \\
\hline
\end{tabular}

Note: The bold font represent statistical significance.

A systematic review showed that good outcome following a ventriculoperitoneal shunt in TBM patients depends on its clinical severity. ${ }^{8}$ It is therefore important to recognize TBM during the early stage. We found that the number of CNS infections causing seizures almost stayed the same, but the percentage was significantly lower during 2014-2017 than during 2010-2013 [48.8\% (59/121) vs $25.4 \%$ (50/197)]. Due to economic development and immunization rate rise, the incidence of meningitis caused by Neisseria meningitidis, Haemophilus influenzae,
Streptococcus pneumoniae meningitis, and vector-borne diseases decreased significantly. ${ }^{9}$ Another possible factor is the availability of new methods during 2014-2017 when more epileptic, immune, or metabolic causes were able to be identified.

Epilepsy has no identifiable cause in about half the people with the condition. The overall prevalence of epilepsy in Chinese children aged $0-19$ years is $2.72 \%{ }^{10}{ }^{10}$ The incidence of childhood convulsive status epilepticus (SE) in developed countries is approximately 10-27/100,000/ year. ${ }^{11}$ A nationwide multicentre study in China revealed that the most common known SE etiology was acute symptomatic etiology $(42.8 \%)$ and $50.2 \%$ of SE was caused by epilepsy of unknown etiology. ${ }^{12}$ Valencia and colleagues stated that EEG is helpful in the great majority of their patients for supporting the diagnosis of epileptic seizures. ${ }^{13}$ The 2014-2017 period included more patients with unknown reason epilepsy (36.5\%) compared to 2010-2013 (19.8\%). This trend is related to the increasing performance of EEG as part of multimodal monitoring in our PICU. There is evidence that poorly controlled seizures in the developing brain negatively impact intellectual functioning. ${ }^{14}$ FIRES is an encephalopathy that could cause severe epilepsy in previously healthy children and adolescents. None of the antiepileptic drugs or anesthetics have decreased the seizure load or reduced the duration of the disease. ${ }^{15}$ The first case of FIRES in China was reported in 2013. The present study included 12 cases

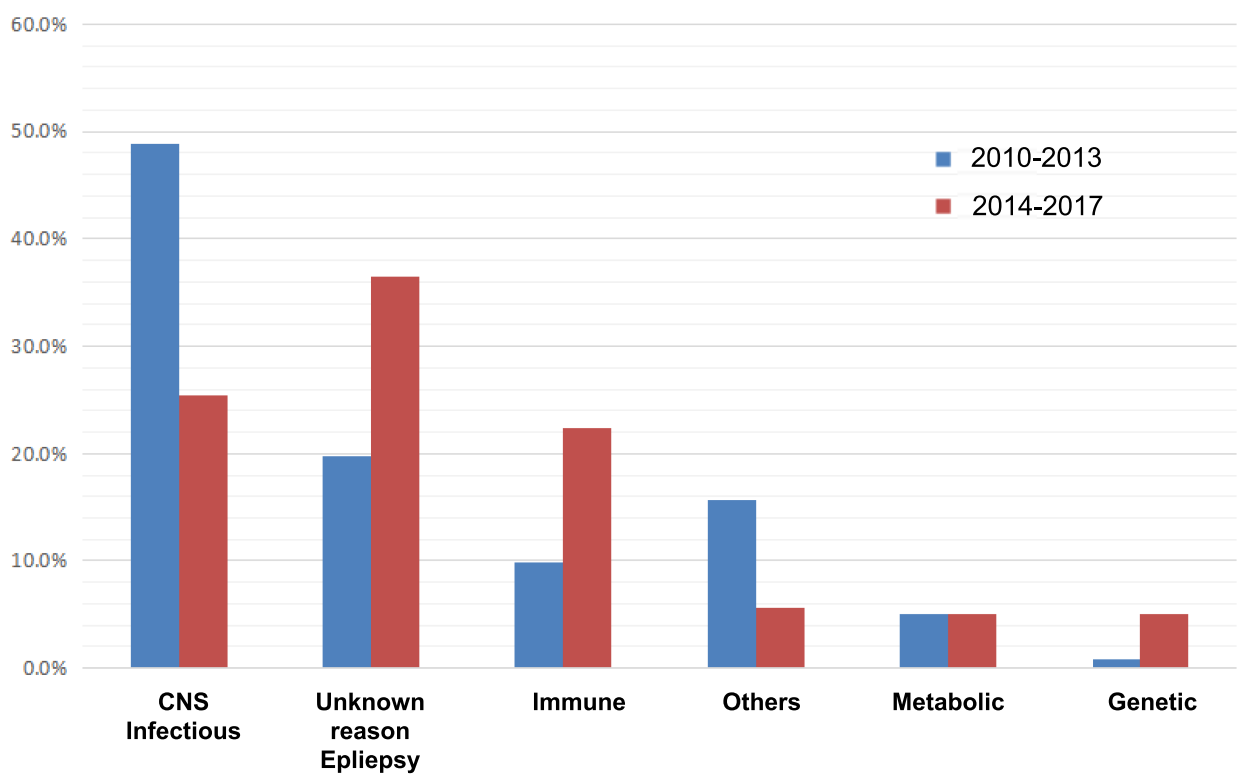

Figure 2 Comparison of etiological causes in patients with seizure in the 2010-2013 $(n=121)$ and 2014-2017 ( $=197)$ periods. 
with FIRES, and 11 cases were observed between 2014 and 2017. We also found that ketogenic diet may be a safe and promising therapy for FIRES and that early initiation of ketogenic diet produces a favorable prognosis. ${ }^{16} \mathrm{EEG}$ could potentially provide real-time bedside monitoring and early electrographic seizure detection, allowing timely intervention that may result in improved outcome. ${ }^{17}$

Encephalitis is a potentially devastating neurological syndrome with different etiologies including direct CNS infection and those mediated by the immune system. AntiNMDAR encephalitis was first described by Dalmau et al in 2007. ${ }^{18}$ Autoimmune encephalitis (AE) is now considered one of the most common causes of noninfectious acute encephalitis. A prospective cohort study by the California Encephalitis Project showed that $65 \%$ of antiNMDAR encephalitis occurred in patients (aged $\leq 18$ years) with encephalitis of uncertain etiology. ${ }^{4}$ The first case of anti-NMDAR encephalitis in China was reported in 2010. Anti-NMDAR antibody test has been available in our institution since 2014. Thus, we found a significant increase in the percentage of immune-related disorders between the 2010-2013 and 2014-2017 periods. All 33 cases of anti-NMDAR encephalitis were found in the 2014-2017 period. Increasing testing for anti-NMDAR encephalitis resulted in frequent identification of cases. Patients with anti-NMDAR encephalitis who receive early immunotherapy might be more likely to completely recover. ${ }^{19}$ We performed a retrospective study of children with anti-NMDAR encephalitis: 44 of the 51 patients (86.2\%) reported good outcomes (score on the modified Rankin scale (mRS) of $0-2)^{20}$

Having an identified cause of epilepsy (genetic, structural, metabolic) was associated with unsatisfactory seizure outcome (48\% drug resistance) and influenced the relative risk associated with other prognostic factors. Dravet syndrome (DS) is one of the most severe epilepsy syndromes of early childhood. Patients with DS experience persistent and severe cognitive and motor deterioration following refractory SE. ${ }^{21}$ Profound status-induced brain edema is a severe, even fatal feature of DS. ${ }^{22}$ In this study, eight cases with DS presented acute encephalopathy after SE and had a poor outcome. Prompt seizure treatment may play a crucial role in the outcome. Seizures may be the first and major presenting feature of an IEM, for example, in a neonate with pyridoxine-dependent epilepsy. ${ }^{23}$ In the present study, IEMs accounted for $5.0 \%$ of all patients. Rapid diagnosis and early specific treatment of metabolic epilepsies due to IEMs are crucial to avoid irreversible sequalae. There could be an antecedent history of developmental delay, specific dietary habits, and associated liver dysfunction. In children with unexplained seizure and coma, especially with liver lesions, cranial MRI basal ganglia lesions, and variable cortex lesions, it is recommended to check blood ammonia, sugar, blood gas, and lactic acid routinely.

There are some limitations to this study. As a retrospective design and selection of PICU patients attended by neurology department physicians in a tertiary center, certain causes of seizure, such as traumatic brain injury, brain tumor, are generally not referred to a pediatrician. Nevertheless, we found a shift in etiologies from infectious diseases towards immune and epileptic origins. These results will be helpful when assessing the underlying etiology of patients with non-traumatic seizures.

\section{Conclusion}

It brings the retrospective of a local population-based study on acute seizure etiology in China.

In conclusion, causes of non-traumatic seizure with impaired consciousness in children include infection, epilepsy, and immune-related disorders. CNS infections are the most common etiology but autoimmune diseases are on the rise. Discovery of new autoantibodies and nextgeneration sequencing could improve our understanding of disease patterns and thereby facilitate early diagnosis and therapeutic management.

\section{Acknowledgments}

This work was supported by the National Key Research and Development Program of China (grant nos. 2016YFC1306202 and 2016YFC0904400) and the National Natural Science Foundation of China (grant nos. 81771409, 81771408, and 81671297).

\section{Disclosure}

The authors report no conflicts of interest in this work.

\section{References}

1. Epilepsy Foundation of America's Working Group on Status Epilepticus. Treatment of convulsive status epilepticus. Recommendations of the Epilepsy Foundation of America's Working Group on Status Epilepticus. JAMA. 1993;270(7):854-859. doi:10.1001/jama.1993.03510070076040

2. Sánchez Fernández I, Jackson MC, Abend NS, et al. Pediatric Status Epilepticus Research Group (pSERG). Refractory status epilepticus in children with and without prior epilepsy or status epilepticus. Neurology. 2017;88(4):386-394. doi:10.1212/WNL.00000000 00003550 
3. Sahin S, Yazici MU, Ayar G, et al. Seizures in a pediatric intensive care unit: a prospective study. J Trop Pediatr. 2016;62:94-100. doi:10.1093/tropej/fmv076

4. Gable MS, Sheriff H, Dalmau J, et al. The frequency of autoimmune N-methyl-D-aspartate receptor encephalitis surpasses that of individual viral etiologies in young individuals enrolled in the California Encephalitis Project. Clin Infect Dis. 2012;54:899-904. doi:10.1093/ cid/cir1038

5. Neligan A, Bell GS, Johnson AL, et al. The long-term risk of premature mortality in people with epilepsy. Brain. 2011;134:388-395. doi:10.1093/brain/awq378

6. Boucher A, Herrmann JL, Morand P, et al. Epidemiology of infectious encephalitis causes in 2016. Med Mal Infect. 2017;47 (3):221-235. doi:10.1016/j.medmal.2017.02.003

7. Glaser CA, Honarmand S, Anderson LJ, et al. Beyond viruses: clinical profiles and etiologies associated with encephalitis. Clin Infect Dis. 2006;43:1565-1577. doi:10.1086/509330

8. Rizvi I, Garg RK, Malhotra HS, et al. Ventriculo-peritoneal shunt surgery for tuberculous meningitis: a systematic review. J Neurol Sci. 2017;375:255-263. doi:10.1016/j.jns.2017.02.008

9. Lundbo LF, Benfield T. Risk factors for community-acquired bacterial meningitis. Infect Dis (Lond). 2017;49(6):433-444. doi:10.1080/ 23744235.2017.1285046

10. Gu L, Liang B, Chen Q, et al. Prevalence of epilepsy in the People's Republic of China: a systematic review. Epilepsy Res. 2013;105:195-205. doi:10.1016/j.eplepsyres.2013.02.002

11. Capovilla G, Beccaria F, Beghi E, et al. Treatment of convulsive status epilepticus in childhood: recommendations of the Italian League Against Epilepsy. Epilepsia. 2013;54:23-34.

12. Wang T, Wang J, Dou Y, et al. Chinese Paediatric Status Epilepticus Task Force. Clinical characteristics and prognosis in a large paediatric cohort with status epilepticus. Seizure. 2020;80:5-11. doi:10.1016/j.seizure.2020.04.001

13. Valencia I, Lozano G, Kothare SV, et al. Epileptic seizures in the pediatric intensive care unit setting. Epileptic Disord. 2006;8:277-284.
14. Fangsaad T, Assawabumrungkul S, Damrongphol P, et al. Etiology, clinical course and outcome of infant epilepsy: experience of a tertiary center in Thailand. J Clin Neurosci. 2019;59:119-123. doi:10.1016/j.jocn.2018.10.117

15. Kramer U, Chi CS, Lin KL, et al. Febrile infection-related epilepsy syndrome (FIRES): pathogenesis, treatment, and outcome: a multicenter study on 77 children. Epilepsia. 2011;52:1956-1965. doi:10.1111/j.1528-1167.2011.03250.x

16. Peng P, Peng J, Yin F, et al. Ketogenic diet as a treatment for super-refractory status epilepticus in febrile infection-related epilepsy syndrome. Front Neurol. 2019;10:423. doi:10.3389/fneur.2019.00423

17. Lai YC. Seizure detection in the PICU: can we "See" Seizures better in color? Pediatr Crit Care Med. 2015;16:486-487. doi:10.1097/ PCC.0000000000000369

18. Dalmau J, Tüzün E, Wu HY, et al. Paraneoplastic anti-N-methylD-aspartate receptor encephalitis associated with ovarian teratoma. Ann Neurol. 2007;61:25-36. doi:10.1002/ana.21050

19. Luca N, Daengsuwan T, Dalmau J, et al. Anti-N-methyl-D-aspartate receptor encephalitis: a newly recognized inflammatory brain disease in children. Arthritis Rheum. 2011;63:2516-2522. doi:10.1002/ art.30437

20. Wang Y, Zhang W, Yin J, et al. Anti-N-methyl-d-aspartate receptor encephalitis in children of Central South China: clinical features, treatment, influencing factors, and outcomes. $J$ Neuroimmunol. 2017;312:59-65. doi:10.1016/j.jneuroim.2017.09.005

21. Tian X, Ye J, Zeng Q, et al. The clinical outcome and neuroimaging of acute encephalopathy after status epilepticus in Dravet syndrome. Dev Med Child Neurol. 2018;60:566-573. doi:10.1111/dmcn.13727

22. Myers KA, McMahon JM, Mandelstam SA, et al. Fatal cerebral edema with status epilepticus in children with dravet syndrome: report of 5 cases. Pediatrics. 2017;139:e20161933. doi:10.1542/ peds.2016-1933

23. Rahman S, Footitt EJ, Varadkar S, et al. Inborn errors of metabolism causing epilepsy. Dev Med Child Neurol. 2013;55:23-36. doi:10.1111/j.1469-8749.2012.04406.x
International Journal of General Medicine

\section{Publish your work in this journal}

The International Journal of General Medicine is an international, peer-reviewed open-access journal that focuses on general and internal medicine, pathogenesis, epidemiology, diagnosis, monitoring and treatment protocols. The journal is characterized by the rapid reporting of reviews, original research and clinical studies across all disease areas. The manuscript management system is completely online and includes a very quick and fair peer-review system, which is all easy to use. Visit http://www.dovepress.com/ testimonials.php to read real quotes from published authors. 